
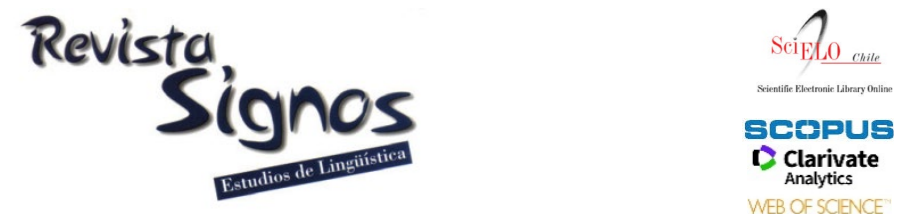

\title{
El rol de la expectativa pragmática de consistencia en la resolución de la referencia definida repetida durante el diálogo*
}

\section{The role of the pragmatic expectation of consistency in the resolution of the repeated definite reference during dialogue}

\author{
Miguel Ibaceta \\ PONTIFICIA UNIVERSIDAD CATÓLICA \\ DE CHLE \\ CHILE \\ maibacet@gmail.com
}

\author{
Nerea Aldunate \\ PONTIFICIA UNIVERSIDAD CATÓLICA \\ DE CHILE \\ CHILE \\ npalduna@uc.cl
}

\author{
Ricardo Morales \\ PONTIFICIA UNIVERSIDAd CATÓLICA DE \\ CHILE \\ CHILE \\ rimorales@uc.cl
}

Francisco Villalón

PONTIFICIA UNIVERSIDAD CATÓliCA DE CHILE

CHILE

villalon.fj@gmail.com
Edmundo Kronmüller ${ }^{1}$

PONTIFICIA UNIVERSIDAD CATÓLICA DE CHILE

CHILE

ekr@uc.cl

Recibido: 07-III-2018 / Aceptado: 29-X-2018

DOI: $10.4067 /$ S0718-09342019000300854

\section{Resumen}

La conversación está guiada por expectativas de comportamiento comunicativo compartidos por los interlocutores. Estas expectativas permiten a los interlocutores interpretar los enunciados y lograr la comprensión mutua. El objetivo del presente estudio es determinar el rol de la expectativa pragmática de consistencia en la resolución de la referencia definida repetida en contextos conversacionales. Para ello, generamos una situación experimental en la que, para resolver la referencia, el oyente debía asumir comportamiento consistente de parte del hablante, pues el ítem lingüístico la dejaba completamente indeterminada. En específico, la referencia contenía una negación del tipo 'el que no es la lentejita'. El experimento manipuló el nivel de consistencia de los hablantes y la presencia o no de 'precedentes referenciales', esto es, acuerdos temporales para nombrar entidades durante el diálogo. Mediante el análisis de los patrones de selección de objetos y los movimientos oculares de los oyentes durante la interpretación, el estudio mostró que los oyentes utilizaban la expectativa de consistencia, y que la integración de esta expectativa en la comprensión sucedía en los primeros momentos de la interpretación de la referencia. En conclusión, la ambigüedad propia del lenguaje puede ser sobrellevada por nuestra sensibilidad a los patrones generales de comportamiento durante un diálogo, y en particular, la consistencia.

Palabras Clave: Procesamiento del lenguaje, diálogo, expectativas pragmáticas, referencia, seguimiento ocular. 


\begin{abstract}
Conversation is guided by expectations of communicative behavior. These expectations allow interlocutors to interpret utterances and reach mutual understanding. The objective of the present study is to determine the role of the pragmatic expectation of consistency in the resolution of repeated definite reference in conversational settings. We generated an experimental situation in which, to resolve reference, listeners had to rely on the expectation of consistency, since the linguistic input left it underdetermined. Specifically, the reference contained a negation of the type 'the object is not the little lentil'. We manipulated the consistency of the speakers and the presence or absence of 'referential precedents'-i.e., temporary agreements to name entities during the dialogue. The analysis of object selection patterns and eye-movements revealed that listeners relied on the expectation of consistency, and that it is integrated at early stages of the interpretation process. In conclusion, the ambiguity of language can be overcome by our sensitivity to general patterns of behavior during dialogue, particularly, consistency.
\end{abstract}

Key Words: Language processing, dialogue, pragmatic expectations, reference, eyetracking.

\title{
INTRODUCCIÓN
}

Una niña ayuda a su madre a arreglar un computador que ha dejado de funcionar. Ya lo han desarmado, han encontrado la falla y se disponen a poner todas las piezas de vuelta en su lugar. Dado que son muchas las piezas y no conocen el nombre para todas, durante el proceso de desarme han inventado algunos nombres que les han permitido organizar el trabajo. Por ejemplo, la madre le ha pedido que guarde 'la lentejita con patas de alambre' o que sostenga 'el ciempiés rectangular'. Ya quedando pocas piezas para poner de vuelta en su lugar, la madre le pide a su hija que le pase 'esa pieza que no es la lentejita'. La hija mira las piezas restantes y ve que solo restan la que han llamado 'el ciempiés rectangular' y otra que no han nombrado previamente. Ante esta situación: ¿Qué pieza le pasará la hija, el objeto sin nombre o el objeto que han llamado 'el ciempiés rectangular'? Claramente, esta es una situación de ambigüedad referencial, dado que, en tanto ninguna de ellas es 'la lentejita', ambas son potenciales referentes.

La referencia definida es un acto ubicuo en toda conversación. Es mediante ella que los interlocutores buscan llevar a un referente específico a la atención conjunta (Tomasello, Carpenter, Call, Behne \& Moll, 2005). La propiedad de ser 'definida' está dada por el hecho que su uso presupone que existe una entidad única y específica que puede ser identificada como referente (Chafe, 1976). Pero más importante aún, es que la 'identificabilidad' y 'unicidad' están circunscritas al dominio contextual compartido entre los hablantes (Clark \& Marshal, 1981). Es decir, una referencia es 'definida' si existe un único referente que puede ser inferido como tal, a la luz del contexto comunicativo compartido entre dos o más interlocutores (Clark, 1996). Una de las maneras en las que un referente puede ser único en un contexto conversacional particular es por medio de un proceso de anclaje (grounding), el que a su vez puede 
implicar el uso repetido de una misma forma lingüística (o similar) para referir a un mismo objeto (Clark \& Brennan, 1991). El concepto de referencia definida repetida captura todas estas características.

Por todo lo anterior, en tanto acto comunicacional, no son las palabras las que refieren, sino los hablantes que ocupan las palabras para ello (Kronmüller, 2008). Entonces, para lograr resolver la referencia conversacional - esto es, identificar al objeto que se quiere traer a la atención conjunta- los oyentes deben ir más allá del significado convencional de las palabras y considerar información relativa a los hablantes (Strawson, 1950). Entre esta información, están las expectativas pragmáticas, que son patrones generales de comportamiento comunicativo que ayudan a los interlocutores a interpretar los enunciados verbales. En general, los interlocutores tienen la expectativa de que las contribuciones del otro serán hechas de manera de facilitar la comprensión para así lograr los objetivos de la conversación (Grice, 1975). Así, apelando a sus expectativas pragmáticas, podríamos hipotetizar que la niña podría inferir que la madre se refiere al objeto que no tiene nombre, pues si la madre hubiese querido referirse al objeto que han llamado 'el ciempiés', entonces lo más fácil y simple sería ser consistente y ocupar ese nombre que ambas conocen y no un enunciado indirecto y complejo como 'esa pieza que no es la lentejita'.

La presente investigación busca evaluar el rol que cumple la expectativa pragmática de consistencia en la resolución de la referencia definida repetida en situaciones como las del ejemplo dado, donde no es posible utilizar otra información que no sea contextual dado que la información lingüística (en el ejemplo 'no la lentejita') no permite identificar el referente de manera unívoca (Kronmüller, Noveck, Rivera, Jaume \& Barr, 2017). En segundo lugar, busca observar en tiempo real el proceso de interpretación para entender en qué momento la expectativa de consistencia tiene su efecto en la comprensión. Para lograr estos objetivos, generamos una situación como la de nuestro ejemplo, utilizando el paradigma experimental de 'mundo visual', el que es ampliamente usado en investigación psicolingüística y que detallaremos en la sección metodológica (Huettig, Rommers \& Meyer, 2011).

\section{Marco teórico}

La conversación es una actividad regulada por expectativas de comportamiento sostenidas entre los interlocutores. El cumplimiento de estas expectativas permite el normal flujo de la conversación y la comprensión mutua. Quizás la expectativa más conspicua y general es la de cooperación, que consiste en esperar del interlocutor una conducta comunicativa que facilite el cumplimiento de los objetivos de una conversación (Grice, 1975). Es esta expectativa y el comportamiento verbal del hablante -esto es, las palabras utilizadas en el enunciado- lo que permite que un oyente pueda inferir lo que un hablante quiere comunicar. Uno de los actos más comunes de una conversación, donde se manifiesta la interdependencia de las 
expectativas pragmáticas y el comportamiento verbal, es la referencia definida repetida, donde una expresión lingüística, normalmente una frase nominal (artículo definido, un sustantivo y un adjetivo), se establece como precedente referencial para volver a referir nuevamente a lo mismo (Barr \& Keysar, 2002; Shintel \& Keysar, 2007; Kronmüller \& Barr, 2015).

Existe amplia evidencia en la psicología del lenguaje que indica que los hablantes son consistentes en la manera en que nombran a las cosas y que esta consistencia ayuda a los oyentes a reconocer más fácilmente cuál es el objeto al que los hablantes se refieren. Por ejemplo, Brennan y Clark (1996) mostraron que cuando el hablante se refiere a un sillón particular como el 'sillón viejo' en una primera instancia, los hablantes seguían llamándolo de la misma manera con posterioridad, incluso en un nuevo contexto donde era el único sillón presente, por lo que el adjetivo ‘viejo' ya no era necesario para distinguir. Esta consistencia también se ha documentado en diversas tareas experimentales en las que se motiva la producción de referencias definidas repetidas, ya sea con tangramas (Horton \& Slaten, 2012; Clark \& WilkesGibbs, 1986), objetos o imágenes extrañas (Barr \& Keysar, 2002), mapas (Knutsen \& Le Bigot, 2017) o laberintos (Garrod \& Anderson, 1987; para un meta-análisis sobre un sub-grupo de esta investigación ver Kronmüller \& Barr, 2015).

Desde el lado del oyente, esta consistencia ayuda a identificar a los referentes de manera más precisa y rápida. Por ejemplo, se ha observado que al escuchar una misma expresión, los oyentes seleccionan los objetos previamente nombrados con el mismo nombre de manera más rápida (Barr \& Keysar, 2002; Metzing \& Brennan, 2003; Kronmüller \& Barr, 2007; Brown-Schmidt, 2009; Horton \& Slaten, 2011). Más aún, esta ventaja del referente ya nombrado puede observarse en los movimientos oculares de los oyentes en fases tempranas $(200 \mathrm{~ms})$ del proceso de interpretación de una frase nominal contenida en una instrucción como 'La lentejita con patas de alambre' (Kronmüller \& Barr, 2015).

La evidencia más directa sobre la influencia de la expectativa de consistencia en el procesamiento de la referencia definida repetida se da en los casos en que un hablante utiliza una expresión diferente para referirse a un objeto ya nombrado. En su influyente estudio, Metzing y Brennan (2003) mostraron que el uso de una expresión nueva para referirse a un objeto previamente nombrado de manera diferente provocaba cierta interferencia que se manifestaba en un retraso en la interpretación por evitar mapear esta nueva forma de referirse al objeto ya nombrado. Esta interferencia sucedía incluso cuando esta nueva expresión fuese tan buen descriptor del objeto como la primera expresión, por ejemplo, al decir 'la cajita negra con pelitos' para referirse al objeto previamente nombrado como 'el ciempiés rectangular'. Nuevamente, los estudios que observaron el proceso de interpretación en tiempo real -mediante el uso de eye-tracker en el paradigma del 'mundo visual'- mostraron que esta 
interferencia ocurría cerca de 200 milisegundos después de escuchar la frase nominal de la instrucción (Kronmüller \& Barr, 2007; Brown-Schmidt, 2009).

Todos los hallazgos aquí expuestos sobre el efecto de la referencia previa sugieren que la consistencia es un elemento que influye en la interpretación de la referencia definida repetida y que su efecto es robusto y sistemático (Kronmüller \& Barr, 2015). Sin embargo, esta evidencia podría ser interpretada por explicaciones alternativas al efecto de la consistencia que no se sustentan en una expectativa pragmática, sino que en 1) un sesgo general de interpretación, o 2) un problema de re-conceptualización. En el primer caso, el retraso en mapear una nueva palabra a un objeto ya nombrado vendría a ser explicado por la tendencia sostenida de no utilizar una palabra diferente para un objeto que ya tiene nombre. Esta tendencia ha sido postulada en el contexto de la adquisición de vocabulario con niños y denominado como 'sesgo de mutua exclusividad' (Mutual Exclusivity Bias) (Markman \& Wachtel, 1988) o el principio de 'nueva palabra-categoría sin nombre' (Novel Name -Nameless Category Principle) (Mervis \& Bertrand, 1994). En el segundo caso, el retraso sería explicado por un problema de reconceptualización, donde la nueva expresión puede gatillar la necesidad de conceptualizar al objeto de una nueva forma (Carmichael, Hogan \& Walter, 1932). Ciertamente, una cosa es un 'ciempiés rectangular' y otra muy distinta es una 'cajita negra con pelitos'.

Como consecuencia de lo anterior, para evaluar el rol de la expectativa pragmática de consistencia en la resolución de la referencia definida repetida es necesario, por un lado, generar una situación en la cual no exista un nombre o descripción que gatille al sesgo mencionado y, también, que no genere una necesidad de reconceptualización. En otras palabras, una situación en la que solo se pueda resolver una ambigüedad referencial basándose en la expectativa de consistencia. Así, en vez de un caso en el que un hablante cambia la expresión ('el ciempiés rectangular') por una nueva ('la cajita blanca con pelitos'), se necesita un caso en el que el hablante utiliza una expresión referencial con negación del tipo 'no la lentejita' y agregar la presencia de otro objeto que no haya sido nombrado previamente (Kronmüller et al., 2017). De esta manera, frente a un objeto sin nombre y uno ya nombrado, la expectativa de consistencia llevaría al oyente a seleccionar al primero, siguiendo el mismo razonamiento que ilustramos en nuestro ejemplo inicial. Pero, adicionalmente a esto, es necesario comparar un caso en el que el hablante es consistente al utilizar los precedentes referenciales con otro en el que a veces el hablante se comporta de manera inconsistente. El diseño experimental de la presente investigación implementa estos casos.

\section{Marco metodológico}

Basándonos en la tarea reportada en Kronmüller et al. (2017), implementamos una situación experimental donde el comportamiento verbal de un hablante deja 
completamente indeterminada la referencia y solo mediante la expectativa de consistencia se pueda resolver la ambigüedad sobre cuál es el objeto referido. En el estudio, un oyente debe seguir las instrucciones de un hablante para seleccionar uno entre tres objetos extraños que no tienen una manera convencional de nombrarlos: un objeto Negado, un objeto Sin nombre y un objeto Competidor (ver Figura 1). En una de las instrucciones, el hablante pide al oyente seleccionar un objeto negando que éste sea uno de los otros dos posibles, por ejemplo, 'no es la lentejita' (donde 'la lentejita' es el objeto Negado). Desde un punto únicamente lingüístico, esta instrucción deja indeterminada la referencia, pues el objeto Sin nombre y el objeto Competidor son potenciales referentes. En base a esta situación, generamos cuatro condiciones experimentales definidas por el estatus del objeto Competidor, esto es, si ha sido nombrado o no (la variable Mención), y por el nivel de consistencia esperada de los hablantes (la variable Consistencia).

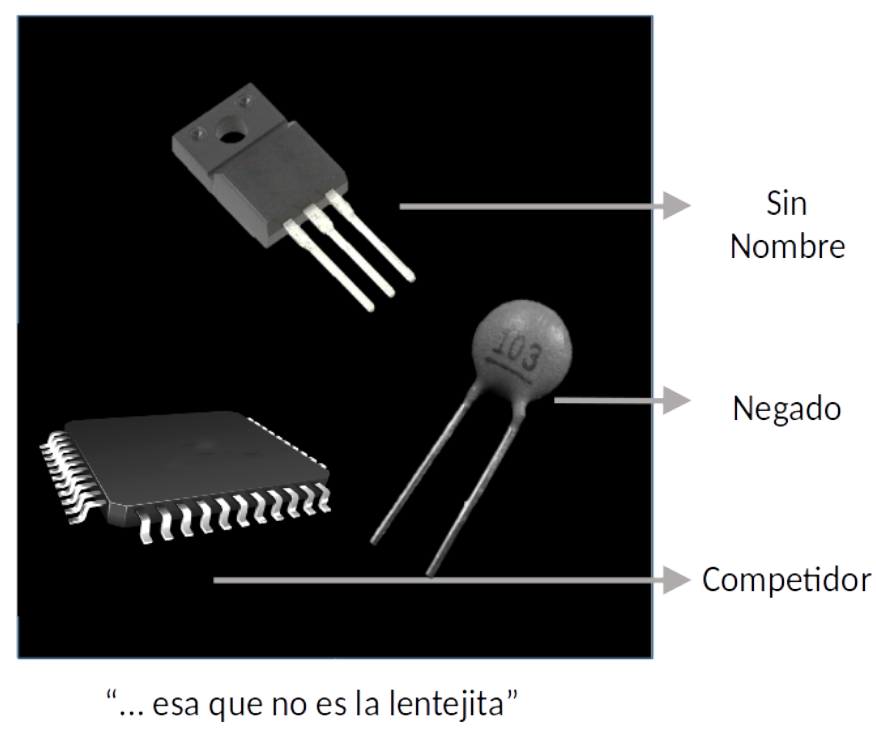

Figura 1. Ejemplo de Ensayo de Testeo.

Pantalla con los tres objetos: Negado, Sin nombre y Competidor. Es en esta situación cuando el participante recibe la instrucción de seleccionar el objeto que no es la 'lentejita'. Lo que sucede previamente a este ensayo configura las distintas condiciones experimentales. (Figura adaptada de Kronmüller et al., 2017).

Así, para dos condiciones experimentales, el objeto Competidor habrá sido mencionado previamente, existiendo así un precedente referencial. Por lo tanto, asumiendo consistencia del hablante, el oyente debiera elegir el objeto Sin nombre, pues, al tener un nombre el objeto Competidor, lo más cooperativo es ser consistente y utilizar ese nombre para referirse a él y no una expresión compleja que contiene una 
negación. En las otras dos condiciones, el objeto Competidor no tendrá un nombre asociado, pues no habrá sido referido con anterioridad. En esta circunstancia, este objeto compite con el objeto Sin nombre, en tanto la consistencia no permite resolver la referencia, siendo cualquiera de los dos objetos potenciales referentes. Junto con esto, manipulamos el nivel de consistencia del hablante. Para un grupo de participantes, el hablante será consistente, en tanto de él cabe esperar que utilice los precedentes referenciales al nombrar repetidamente un objeto. De este modo, con un hablante consistente se debiera dar el patrón de respuesta antes descrito tanto para cuando el objeto Competidor ha sido mencionado previamente, como cuando no lo ha sido. Para la otra mitad de los participantes, el hablante será inconsistente, en tanto no se espera que mantenga los precedentes referenciales. Esta manipulación la implementamos al decirle a los participantes que el hablante padecía la enfermedad de Alzheimer, lo que lo llevaría, a veces, a no recordar la manera en que nombró los objetos previamente. Así, a diferencia del hablante consistente, cuando el objeto Competidor ha sido nombrado, la preferencia por el objeto Sin nombre debiera desaparecer, pues no es seguro que el hablante lo recuerde, dejándolo, para efectos prácticos, sin un nombre, al igual que el objeto Sin nombre. Finalmente, y considerando el perfil temporal de la interpretación de una referencia con negación reportado en Kronmüller et al. (2017), el patrón de preferencias antes descrito debiera observarse, al menos, al finalizar la instrucción que contiene esta negación.

Para analizar el proceso de interpretación en sus etapas más tempranas y a medida que se desarrolla -y que eventualmente desemboca en el patrón de selección reportado-, además de registrar la elección final de los participantes, nos focalizaremos en los movimientos oculares. Los movimientos oculares permiten observar en tiempo real el proceso de interpretación, y por esto permiten identificar el momento en que comienza a tener un efecto la expectativa de consistencia en la resolución de la referencia en caso de ambigüedad. Específicamente, monitoreamos los movimientos oculares para determinar el momento en que la preferencia por el objeto Sin nombre por sobre el objeto Competidor comienza a manifestarse en los patrones de mirada cuando el hablante es consistente y el objeto competidor ha sido mencionado. En contraste, este patrón no debiera observarse para las otras tres condiciones. Esta técnica para investigar el proceso de interpretación en tiempo real se denonima 'mundo visual' (para una revisión ver Huettig et al., 2011) y ha demostrado ser útil para el estudio de la influencia diferentes fuentes de información en la resolución de expresiones referenciales ambiguas. Por ejemplo, se ha estudiado la integración de información fonológica para desambiguar palabras temporalmente ambiguas, como 'cama' y 'casa', donde solo a partir de la segunda sílaba es posible asignar unívocamente la referencia (Allopena, Magnuson \& Tananhaus, 1998). Del mismo modo, se ha estudiado la influencia del contexto pragmático para desambiguar expresiones completamente ambiguas como 'el triángulo' frente a un dominio de posibles referentes que contiene dos triángulos de distintos colores, uno que es 
conocido por el hablante y otro que no lo es (Hanna, Tanenhaus \& Trueswell, 2003). En estos casos, se ha visto que la proporción de miradas al triángulo que es parte del conocimiento compartido entre en hablante y el oyente aumenta rápidamente a medida que se interpreta la palabra 'triángulo'. Dado que lo que se quiere observar en la presente investigación es la influencia de una expectativa pragmática en la interpretación de una expresión referencial ambigua, la técnica del 'mundo visual' es la apropiada, y en específico, la medición del incremento o disminución, en el tiempo, en la proporción de miradas a los distintos objetos que son potenciales referentes.

\subsection{Participantes}

Sesenta estudiantes universitarios chilenos de la Pontificia Universidad Católica de Chile (Mujeres $=24$; edad promedio $=21,2$, entre 18 y 26 años, $\mathrm{SD}=1,9$ ) y hablantes nativos del español, participaron voluntariamente en el experimento a cambio de una retribución de 5.000 pesos. Los estudiantes fueron reclutados mediante avisos distribuidos en el campus y, previo a su participación, firmaron un consentimiento informado acorde a la declaración de Helsinki aprobado por el Comité de Ética Institucional. Los datos de un participante no pudieron ser analizados debido a un error en la recolección de datos en esa sesión.

\subsection{Diseño de investigación}

El experimento implementa un diseño factorial $2 \times 2$ mixto. La variable Consistencia tiene dos condiciones administradas intersujeto: hablante Consistente e Inconsistente. El hablante Consistente corresponde a la voz de un adulto y el hablante Inconsistente a un adulto mayor con indicios tempranos de la enfermedad de Alzheimer. La variable Mención fue administrada intersujeto y sus dos niveles son: Mencionado, en el cual un objeto Competidor ha sido nombrado y No-Mencionado, en el cual este objeto no ha sido mencionado previamente.

\subsection{Procedimiento}

Los participantes tomaron el rol de oyente en una versión del juego de comunicación referencial (Krauss \& Weinheimer, 1964). La tarea consistía en seleccionar un objeto entre tres presentados en la pantalla de un computador, siguiendo las instrucciones de un hablante. En este caso, dada las necesidades de controlar los estímulos, las instrucciones fueron pre-grabadas y presentadas a los participantes a través de parlantes. Para justificar lo anterior, se le hizo creer a los participantes que, dada las dificultades de contar repetidamente con un paciente con Alzheimer (o con un adulto según la condición), se decidió grabar las instrucciones que éste le dio anteriormente a otro participante en la misma tarea que ellos realizarían (la Figura 2 describe la situación experimental). Los objetos carecían de un nombre conocido, ya que se trataba de objetos extraños o partes difícilmente reconocibles de 
otros objetos (ver Figura 1). El uso de objetos extraños obliga a generar precedentes referenciales para poder llevar a cabo la tarea.

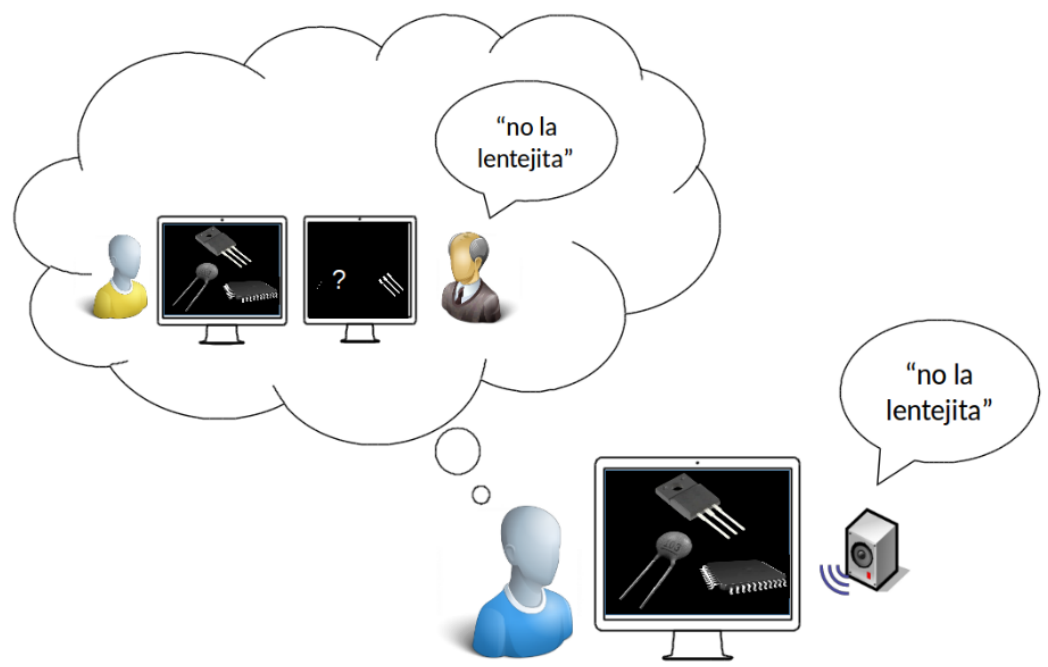

Figura 2. Situación experimental.

El participante está sentado frente al computador, recibiendo las instrucciones sobre qué objeto seleccionar, las que le son entregadas a través de un parlante. El participante es llevado a creer que las instrucciones fueron grabadas previamente por un adulto que participó en el experimento. El participante cree que, al grabar las instrucciones, el adulto solo veía dos de los tres objetos que aparecen es su pantalla. Esto último es para evitar que la expresión 'no es la lentejita' sea considerada como poco cooperativa, lo que sería el caso si el adulto estuviese viendo tres objetos. (Figura adaptada de Kronmüller et al., 2017)

En total, el experimento consistió en 24 ítems, cada uno compuesto de 9 ensayos agrupados en dos etapas. La Figura 3 muestra un esquema de un ítem experimental. Cada ensayo corresponde a una pantalla con tres objetos y una instrucción. Los primeros seis ensayos (tipo A, B y C) corresponden a la etapa de anclaje, en la cual se establecieron los nombres para algunos de los objetos y, además, donde se realizaron las manipulaciones de las dos variables independientes. La manipulación para la variable Mención se realizó en los ensayos B1 y B2, mientras que la manipulación de la variable Consistencia se realizó en los ensayos C1 y C2. Los ensayos de esta etapa fueron randomizados parcialmente, con la restricción de que siempre el ensayo 1 de cada tipo (A, B o C) fuera presentado previamente a los ensayos 2. Los últimos tres ensayos corresponden a la etapa de testeo, pues es en esta etapa donde se encuentra el Ensayo Crítico, del cual provienen los datos para testear nuestras hipótesis. Los otros dos ensayos se incluyeron con el objetivo de encubrir al Ensayo. En esta etapa los ensayos fueron completamente randomizados. La etapa de anclaje sucedía antes que la 
etapa de testeo, sin embargo, no había indicación del cambio de etapa para los participantes, por lo que era percibido como un continuo.

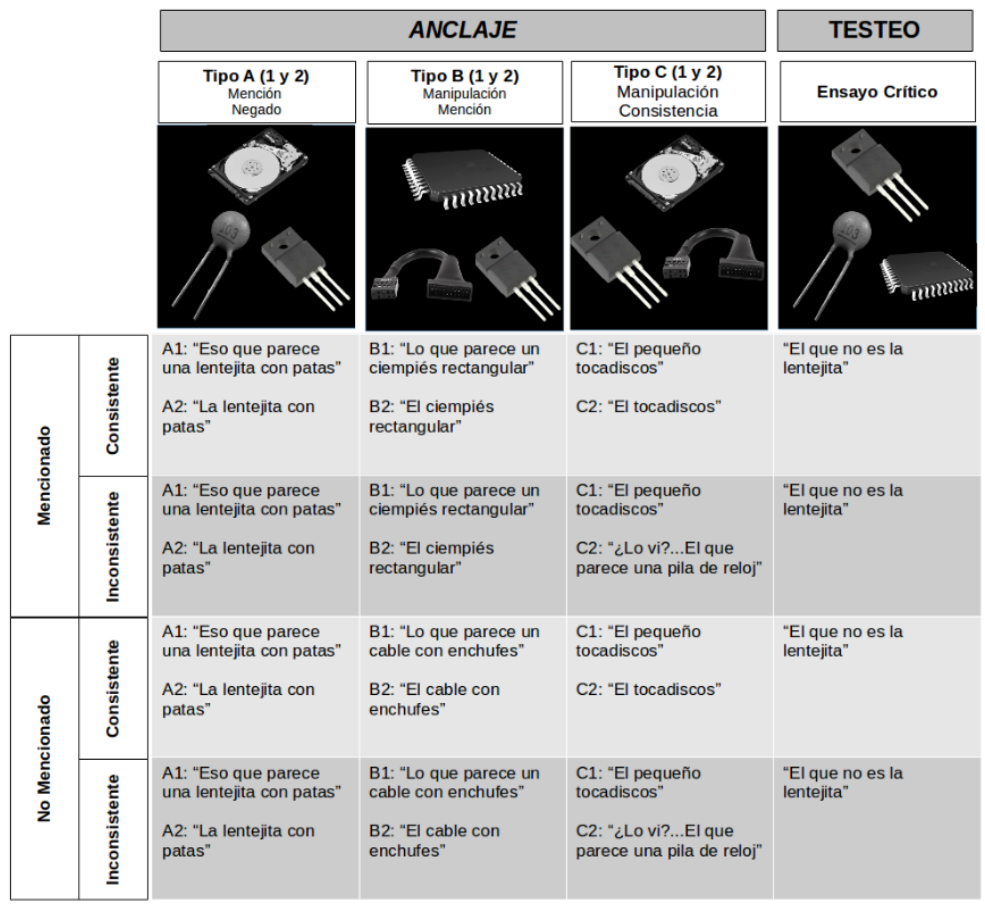

Figura 3. Ejemplo de ítem experimental.

Cada ítem consistió en nueve ensayos divididos en dos fases: anclaje y testeo. En los ensayos tipo A se estableció el precedente referencial para el objeto negado. En el tipo B se generó la manipulación de la variable Mención. Específicamente, se generó un precedente referencial para las condiciones de Mencionado, pero no para las de No Mencionado. En los ensayos tipo C se manipuló la consistencia del siguiente modo: el hablante consistente establecía un precedente referencial para uno de los objetos y lo utilizaba para referir nuevamente al mismo objeto. Por el contrario, el hablante inconsistente utilizaba dos nombres distintos para referirse al mismo objeto ("pequeño tocadiscos' y 'pila de reloj'). Junto con esto, daba indicios de dificultad para recordar al decir ‘¿lo vi?...'. Además, dos ensayos (no representados en la figura) fueron incorporados a la fase de testeo con el propósito de ocultar la naturaleza de la manipulación y evitar la predicción de la posición del Ensayo Crítico.

La Figura 1 muestra un ejemplo de Ensayo Crítico. Es en este ensayo donde se utiliza una expresión con negación y donde aparecen los tres objetos que configurarán la situación descrita arriba: El objeto Negado, Sin nombre y Competidor. Así, frente a la instrucción 'no la lentejita', los posibles referentes son el objeto Sin nombre y el Competidor. Como se dijo, lo que sucede en la etapa de anclaje define las cuatro 
condiciones experimentales. En la condición Consistente Mencionado, el objeto Competidor fue nombrado dos veces en los ensayos tipo B como 'el ciempiés rectangular' por el hablante que consistentemente nombró, en los ensayos tipo C, a un objeto como 'el pequeño tocadiscos'. En la condición Consistente No-Mencionado, el hablante también era consistente en los ensayos $\mathrm{C}$, pero el objeto Competidor no fue nombrado en los ensayos B, sino que en su remplazo fue nombrado otro objeto 'el cable con enchufes'. En la condición Inconsistente Mencionado, el objeto Competidor es nombrado dos veces como 'el ciempiés rectangular'; pero en los ensayos C, el hablante se muestra inconsistente al nombrar a un mismo objeto como 'el pequeño tocadiscos' en C1, pero en C2 muestra duda y signos de pérdida de memoria y le cambia el nombre diciendo: ‘¿Lo vi?... el que parece una pila de reloj'. Esta inconsistencia es explicada al haber presentado al hablante como un adulto con Alzheimer. Finalmente, en la condición Inconsistente No-Mencionado, el hablante se comporta de la misma manera en los ensayos tipo C, pero el objeto competidor no es referido en los ensayos tipo B. Es importante puntualizar que se usaron los mismos archivos de audio, pero en combinaciones diferentes de manera de generar las distintas condiciones experimentales. Para el Ensayo crítico, fue el mismo archivo para todas las condiciones experimentales.

Con el objetivo que las instrucciones siempre fueran consideradas como cooperativas, le fue dicho al participante que, al momento de grabar las instrucciones, el hablante veía solo dos de los tres objetos que aparecían en su pantalla (la del participante) y que su tarea era también determinar cuál de los objetos, el Sin nombre o el Competidor, era el que no aparecía en la pantalla del hablante. De este modo, desde el punto de vista del hablante, la instrucción 'no la lentejita' solo podía referir a un objeto y era por lo tanto plenamente cooperativa.

Mientras el participante seguía las instrucciones se registraron sus movimientos oculares y la selección de objetos que hacía con el mouse del computador. Los participantes demoraron aproximadamente 45 minutos en completar la sesión.

\subsection{Materiales y aparato de registro}

Las imágenes consistían en fotografías en blanco y negro de objetos o partes de objetos extraños difíciles de reconocer que fueron extraídos de internet. Las instrucciones fueron grabadas y luego alteradas para simular la voz de un adulto mayor. Cada instrucción correspondía a un archivo de audio diferente. Un computador controló la presentación de los estímulos visuales y auditivos, sincronizando los eventos con la información recolectada por un eye-tracker. Solo el ojo izquierdo de cada participante fue registrado usando un eye-tracker Eyelink 1000 (SR Research) a una frecuencia de muestreo de $500 \mathrm{~Hz}$. 


\subsection{Análisis}

Para el testeo de nuestras hipótesis, realizamos dos análisis sobre: (a) la proporción de selección final del objeto Sin nombre, y (b) la proporción de tiempo de mirada hacia el objeto Sin nombre con respecto al objeto Competidor. Para ambos utilizamos un modelo lineal mixto, con Consistencia y Mención como factores fijos, y Sujetos e Ítems como factores aleatorios cruzados (Baayen, Dadvidson \& Bates, 2008). Para la primera, dado que es una variable dicotómica (selecciona o no el objeto Sin nombre), utilizamos una distribución binomial; mientras que para la segunda, utilizamos una distribución normal. A continuación explicamos cómo calculamos esta última medida.

Para analizar la proporción de tiempo de mirada hacia el objeto Sin nombre con respecto al objeto Competidor, calculamos, en primer lugar, la proporción de tiempo de mirada para cada objeto. Para el cálculo de estas proporciones, se dividió el tiempo total de la ventana de interés $(1000 \mathrm{~ms})$ para cada ensayo en pequeñas ventanas de 50 milisegundos (bins). Dado que utilizamos una frecuencia de muestreo de $500 \mathrm{~Hz}$, cada una de estas ventanas contenía 25 muestras con un valor categórico que correspondía al área de interés a la cuál la mirada estaba dirigida. Para el caso específico de este experimento, las áreas de interés fueron el objeto Negado, el Competidor y el Sin nombre. Además, se consideró como 'Otro' a una muestra en donde el participante estaba mirando a otro lado, estaba en medio de una sacada o por una pérdida de datos por un pestañeo. Así, para cada ensayo se obtuvo una sucesión de ventanas de 50 milisegundos con la proporción de muestras en cada área de interés. El promedio de esta medida agregada por sujeto se muestra en la Figura 4 (para una explicación de esta manera de analizar datos de movimientos oculares en el contexto de la técnica del 'mundo visual', ver Tanenhaus \& Trueswell, 2005). Finalmente, se realizó una resta entre la medida calculada para el objeto Sin nombre y el Competidor, pues es esta diferencia la que permite testear nuestras hipótesis: una diferencia mayor que cero indica una preferencia hacia el primero y una diferencia menor que cero, hacia el segundo; mientras que, si la substracción es cero, no habría diferencias entre ambos objetos.

Los análisis de la proporción de selección a cada objeto y de la diferencia en la proporción de tiempo mirando al objeto Sin nombre y Competidor, se realizaron comparando el modelo estadístico completo, esto es, con ambas variables independientes y su interacción, con el modelo restringido, esto es, uno que no contuviese el predictor de interés. El resultado de esa comparación se expresa con el estadístico chi-cuadrado (Baayen et al., 2008). Los análisis fueron realizados con $\mathrm{R}$ (versión 3.2.3) y con el paquete lme4 (Bates, Maechler, Bolker \& Walter, 2014). 


\section{Resultados}

\subsection{Selección}

La Tabla 1 muestra el porcentaje de selección de cada uno de los objetos en cada condición experimental. En primer lugar, se observó una tasa baja de selección del objeto Negado en todas las condiciones, lo que es esperable dado que era justamente el objeto que se descartaba como referente. Es interesante notar, sin embargo, una diferencia numérica entre el hablante Consistente y el Inconsistente, donde el objeto Negado fue seleccionado un poco más frecuentemente en el Inconsistente. Para evaluar nuestras predicciones, el contraste relevante es entre la selección del objeto Sin nombre en las condiciones de Mencionado y No-Mencionado para ambos hablantes. En congruencia con nuestras hipótesis, la selección del objeto Sin nombre fue mayor en la condición Consistente Mencionado (63\%) que en la condición Consistente NoMencionado (53\%) (chi-cuadrado(1) $=9.357, \mathrm{p}=0.002$ ), pero no estadísticamente diferente para ambas condiciones del hablante inconsistente (47\% y $43 \%$ para Mencionado y No-Mencionado correspondientemente) $(\operatorname{chi}-\operatorname{cuadrado}(1)=0.975, \mathrm{p}=$ $0.324)$.

Tabla 1. Selección final. Porcentaje de selección de cada objeto en cada una de las condiciones experimentales.

\begin{tabular}{|l|c|c|c|c|}
\cline { 2 - 5 } \multicolumn{1}{c|}{} & \multicolumn{2}{c|}{ Consistente } & \multicolumn{2}{c|}{ Inconsistente } \\
\cline { 2 - 5 } \multicolumn{1}{c|}{} & Mencionado & No-Mencionado & Mencionado & No-Mencionado \\
\hline Competidor & $35 \%$ & $44 \%$ & $45 \%$ & $50 \%$ \\
\hline Negado & $2 \%$ & $3 \%$ & $8 \%$ & $7 \%$ \\
\hline Sin Nombre & $63 \%$ & $53 \%$ & $47 \%$ & $43 \%$ \\
\hline
\end{tabular}

\subsection{Movimientos oculares}

Tras la presentación de los objetos, se observó una disminución de las miradas al objeto Negado concurrente con el incremento de miradas a los objetos Sin nombre y Competidor (ver Figura 4). Al ver las diferencias entre condiciones, se observó que las líneas que representan las miradas a estos objetos divergen solo en la condición Consistente Mencionado. En las otras tres condiciones, el tiempo mirando estos objetos es similar hasta el final de la ventana de observación. 

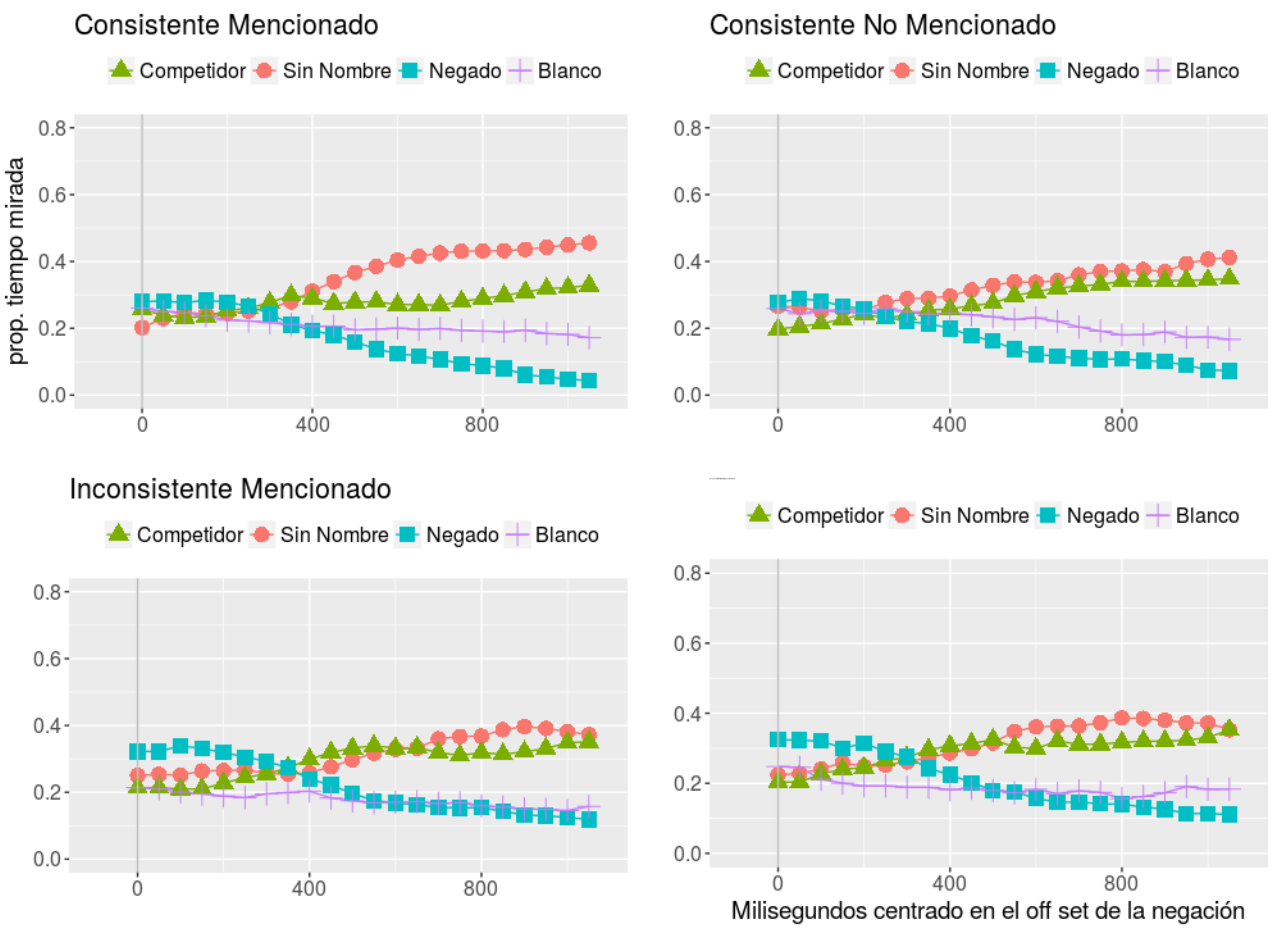

Figura 4. Proporción de tiempo de mirada a cada objeto en cada condición experimental.

Proporción promedio del tiempo de mirada a cada objeto por condición experimental en los primeros 1000 milisegundos posteriores al final de la expresión 'no la lentejita', agrupados en ventanas de 50 milisegundos cada uno.

Según nuestras hipótesis, la pendiente para la condición Consistente-Mencionado debería ser positiva y distinta a la de la condición Consistente-No Mencionado, mientras que tal diferencia en pendientes no debería observarse en las otras dos condiciones; esto es, cuando el hablante es inconsistente (ver Figura 5). En concordancia con estas hipótesis, el análisis estadístico muestra que el componente lineal del modelo (esto es la pendiente), es distinta entre las condiciones Mencionado y No-Mencionado para el hablante Consistente (chi-cuadrado(1) $=19.496, \mathrm{p}<0.0001$ ), pero no para el hablante Inconsistente (chi-cuadrado $(1)=1.033, \mathrm{p}=0.309$ ). 

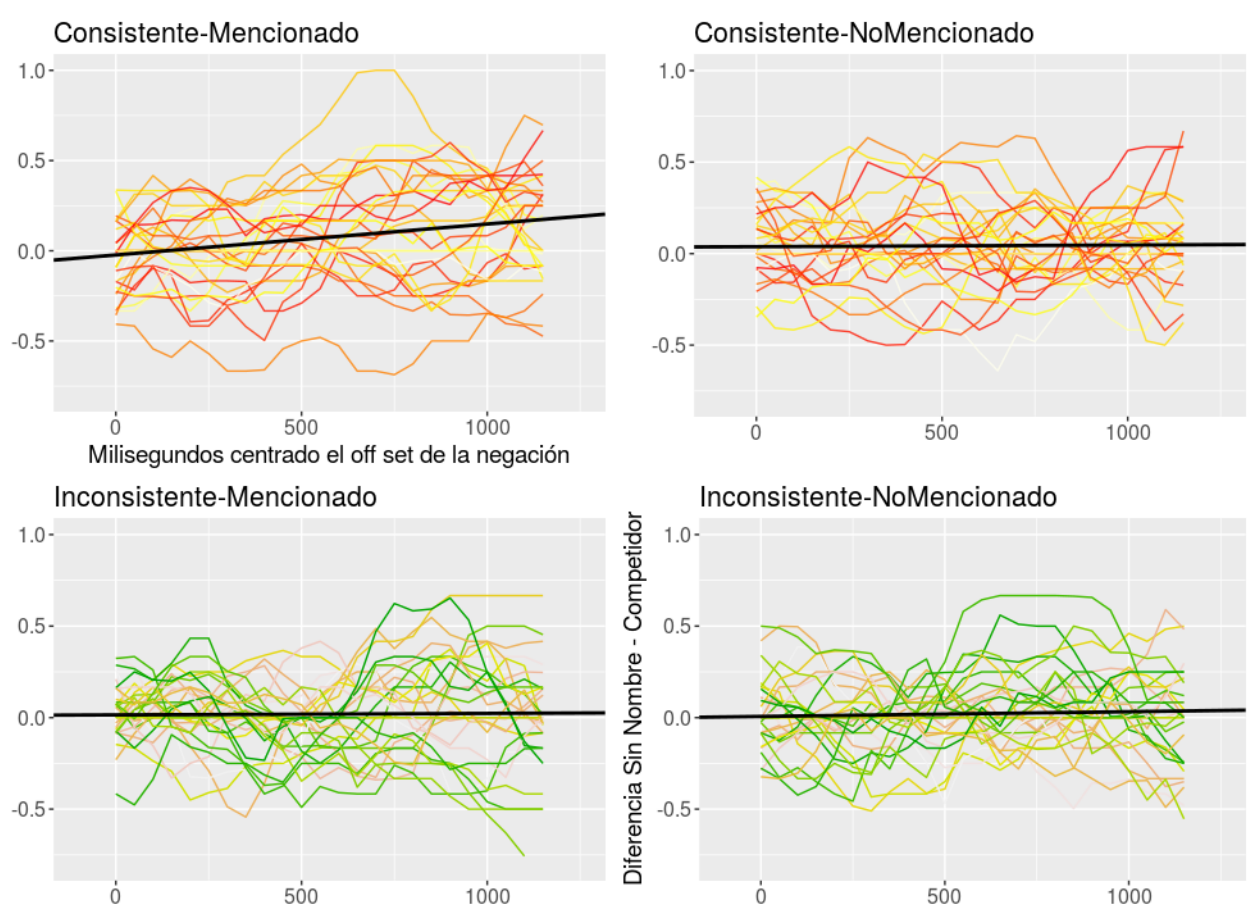

Figura 5. Diferencia entre la proporción de tiempo de mirada al objeto Sin nombre y Competidor por sujeto.

Diferencia entre la proporción promedio de tiempo de mirada al objeto Sin nombre y Competidor por participante en cada una de las condiciones experimentales en los primeros 1000 milisegundos posteriores al final de la expresión 'no la lentejita', agrupados en ventanas de 50 milisegundos. La línea superpuesta corresponde a la línea de regresión calculada para el promedio de las trayectorias individuales.

\section{Discusión}

El presente estudio buscó determinar el rol de la expectativa pragmática de consistencia en la resolución de la referencia definida repetida en circunstancias donde el estímulo lingüístico dejaba completamente indeterminada la referencia. Para ello generamos una situación experimental en la que los participantes debían seguir instrucciones para seleccionar objetos en la pantalla del computador. Una de las instrucciones tenía la forma de una negación, i.e. 'no la lentejita', frente a lo cual el participante solo podía identificar algún referente utilizando información contextual, específicamente la expectativa pragmática de consistencia. Un grupo de participantes siguió las instrucciones de un hablante consistente y el otro de un hablante inconsistente. Por medio de la implementación del paradigma experimental de 'mundo visual', pudimos observar que los oyentes descansan en la expectativa de consistencia para interpretar la referencia definida repetida y que esto se observa desde los 
primeros momentos de la interpretación (en los primeros 1000 milisegundos luego del final de la expresión referencial).

Los resultados obtenidos en este estudio sugieren que la expectativa de que el hablante será consistente y utilizará los precedentes para referirse más de una vez a un objeto, permite a los participantes resolver la ambigüedad producida al utilizar una expresión con negación que deja completamente indeterminada la referencia desde un punto de vista exclusivamente lingüístico. Así, los participantes interpretan que el objeto referido es un objeto no nombrado previamente. Sin embargo, al no poder descansar en esta expectativa - por interactuar con un hablante inconsistente-, un objeto ya nombrado tiene, para efectos de la comunicación, el mismo estatus que un objeto sin nombre. Frente a esto, todo objeto -nombrado o no- es un potencial referente, quedando la referencia sin resolverse. Estos patrones se observan en la selección de los participantes y puede rastrearse hasta los primeros momentos de la interpretación: durante el primer segundo del final de la expresión referencial.

\subsection{Implicancias para la literatura}

Los resultados de esta investigación permiten descartar explicaciones alternativas al efecto de los precedentes referenciales en la resolución de la referencia definida repetida que no se basan en la pragmática de la comunicación. Estas explicaciones alternativas se sustentan en el 'sesgo de mutua exclusividad' (Mutual Exclusivity Bias) (Markman \& Wachtel, 1988), el principio de 'nueva palabra -categoría sin nombre' (Novel Name-Nameless Category Principle) (Mervis \& Bertrand, 1994), y en las dificultades de reconceptualización (Carmichael et al., 1932). En efecto, al no haber un nuevo nombre involucrado por el uso de la referencia con negación, no es posible apelar a ninguno de los sesgos y tampoco a problemas de reconceptualización. En conclusión, la tendencia a evitar una nueva forma lingüística para referir a un objeto, ya sea con una nueva expresión o con una negación, es un efecto pragmático basado en expectativas de comportamiento comunicativo entre los interlocutores. Este efecto tiene un claro perfil temporal que va desde los primeros momentos de la interpretación hasta la resolución definitiva del acto referencial.

En los últimos años ha habido un renovado interés por el estudio de los fenómenos pragmáticos en la comunicación desde una perspectiva experimental (Kronmüller, Morisseau \& Noveck, 2014; Noveck, 2018; Noveck \& Reboul, 2008; Noveck \& Sperber, 2004). Parte importante de este interés pasa por testear predicciones que pueden derivarse de aproximaciones normativas a la comunicación y el uso del lenguaje, en particular, las propuestas de Grice (1957, 1975). Si bien Grice no pretendió describir el cómo se produce la comprensión mutua durante una conversación, entregó elementos para generar descripciones susceptibles de ser testeadas empíricamente. Esto último ha sido facilitado por el advenimiento de técnicas experimentales como el seguimiento de los movimientos oculares durante el 
procesamiento del lenguaje y el paradigma del 'mundo visual'. En conjunto, lo anterior ha permitido superar uno de los desafíos más importantes de la investigación del lenguaje en uso: llevar el diálogo al laboratorio (Brennan \& Hanna, 2017; Tananhaus $\&$ Truewell, 2005). Es en el contexto del estudio del lenguaje en uso donde la presente investigación hace su contribución.

En primer lugar, los resultados de este trabajo dan cuenta de que el procesamiento del lenguaje durante en diálogo es flexible, en tanto se adapta a las particularidades de cada interacción comunicativa (Brennan, Galati \& Kuhlen, 2010; Kronmüller, 2008). En efecto, si bien cabe esperar de los hablantes que sean consistentes, esto no necesariamente produce procesos rígidos de interpretación. Así, la presente investigación muestra cómo los oyentes se pueden adaptar a las características del hablante en la comprensión de la misma manera en que los hablantes se adaptan a las características de los oyentes como, por ejemplo, cuando se habla con un niño o con una persona no nativa del propio lenguaje. En segundo lugar, esta adaptación se produce desde los primeros momentos de la interpretación, mostrando que el sistema no pierde la velocidad que es necesaria para satisfacer las demandas naturales de una conversación, en específico, las impuestas por la dinámica de turnos, que obliga a los participantes a producir sus enunciados de manera contingente a los enunciados del otro. Muchas veces la dinámica de turnos muestra sobreposición entre el momento en que uno de los participantes termina su turno y el otro lo toma (Levinson \& Torreira, 2015), lo que se ha mostrado que es el caso en distintas culturas, con algunas variaciones (Enfield, Stivers \& Levinson, 2010).

En tercer lugar, un aspecto del lenguaje en uso que ha sido fuente de debate en los últimos años es el rol del conocimiento compartido y sabido como compartido o 'piso común' (common ground) en la interpretación de la referencia conversacional. Una de las primeras propuestas fue que éste era el contexto intrínseco de la comunicación (Clark \& Carlson, 1981; Clark, 1996). Así, al interpretar una referencia definida, un oyente debía restringirse a los potenciales referentes que son compartidos con el hablante. Ciertamente, un hablante no puede referir a algo que no sabe que existe, por lo tanto, los referentes no conocidos por el hablante no deberían ser considerados por el oyente, aun cuando la descripción dada se aplique perfectamente. Por ejemplo, un oyente no debiera interpretar como referente de la expresión 'el libro' a un libro que él sabe que el hablante no está en conocimiento (es conocimiento privado del oyente). Sin embargo, sabemos que muchas veces los oyentes no se restringen al piso común, generando interpretaciones 'egocéntricas' que pueden potencialmente causar malos entendidos; como considerar un libro que el hablante no sabe que existe como referente de 'el libro’ (Keysar, Barr, Balin \& Brauner, 2000).

Esta focalización en el estudio del rol del piso común en la referencia definida no ha permitido avanzar del mismo modo en otros aspectos relacionados con la pragmática de la comunicación y que no necesariamente se relacionan con éste. Un 
ejemplo son las expectativas pragmáticas generales que hemos examinado aquí, y que se basan en patrones recurrentes de comportamiento comunicativo. Al basarse en patrones generales no están relacionados un un hablante en particular y en consecuencia, con el piso común (Levinson, 2001). La expectativa de consistencia puede ser una de ellas (Shintel \& Keysar, 2007). Si bien el presente estudio no permite pronunciarse sobre la relación entre el piso común y expectativa general de consistencia, estudios previos apuntan en esa dirección (Kronmüller et al. 2017; Kronmüller \& Barr, 2015).

Finalmente, el presente estudio puede enmarcarse dentro de la investigación más amplia de la referencia anafórica en lingüística y psicolingüística (Huang, 2007), que ha abordado dimensiones que van desde su uso en el procesamiento del lenguaje natural por medio de inteligencia artificial (Sukthanker, Poria, Cambria \& Thirunavukarasu, 2018) hasta sus correlatos neurales (Nieuwland \& van Berkum, 2008; Osterhout \& Mobely, 1995). En el estudio de la comprensión de textos escritos, la anáfora puede entenderse como una referencia a entidades mencionadas previamente (Mitkov, 1999) que establece una coherencia discursiva al producir un enlace referencial mediante el uso de razonamiento de tipo inferencial (Streb, Hennighausen \& Rösler, 2004). Dos mecanismos complementarios se han asociados a la identificación de un referente en el uso de una anáfora. Gernsbacher (1989) sostiene que para que una persona logre correctamente identificar el referente de una expresión anafórica debe intensificar, o incrementar, la activación de potenciales referentes y suprimir, o disminuir, la activación de otros. Este proceso descansaría en factores morfológicos, lexicales, sintácticos, contextuales, así como también en el conocimiento del mundo (Mitkov, 2009). De manera similar, la resolución de la ambigüedad referencial en el diálogo que se refleja en los resultados de la presente investigación descansaría también en un proceso inferencial de razonamiento sustentado por expectativas de comportamiento comunicativo, lo que no es sino conocimiento de mundo. En efecto, las aproximaciones pragmáticas a la anáfora (Huang, 2000) postulan que ésta se resuelve a partir de un razonamiento heurístico basado en principios neo-griceanos (Levinson, 2001; Cornish, 2008).

\subsection{Limitaciones y futuras investigaciones}

El presente estudio entrega evidencia sobre el rol de la expectativa pragmática de consistencia durante la comunicación referencial y también del nivel de flexibilidad en el uso de estas claves comunicativas durante la interpretación. Una manera de avanzar en el estudio de esta flexibilidad es mediante la implementación de diseños inter sujeto. En efecto, el presente estudio muestra una adaptación a la situación comunicativa, pero no necesariamente una adaptación al hablante. Para indagar en esto último son necesarios diseños intra sujeto, esto es, una situación experimental en 
la que un mismo oyente interactúe con dos hablantes, uno consistente y otro inconsistente.

Una dimensión a evaluar en futuras investigaciones tiene relación con las diferencias individuales. En efecto, en este tipo de investigaciones, donde se aborda el desafío de llevar la conversación al laboratorio, se observa variabilidad en la respuesta de los sujetos. Esta variabilidad bien podría explicarse por la diferencia en habilidades comunicativas y habilidades sociales de los participantes (Baron-Cohen, 1988). La incorporación de mediciones de estas características podría contribuir a explicar la variabilidad encontrada en el desempeño de la tarea de comunicación referencial y, además, a arrojar luces sobre el rol de estas habilidades generales en el proceso de lograr la comprensión mutua durante una conversación.

Sumado a lo anterior, el hecho de que la manipulación experimental para generar la condición de inconsistencia se haya basado en hablantes con Alzheimer deja abierta la pregunta de si, por tratarse de una situación que no necesariamente es familiar, están influyendo factores que pueden generar otros sesgos, como los afectivos (Pyszcynki \& Greenberg, 1987). Esto conlleva la necesidad de observar lo que ocurriría de forma más cotidiana en diferentes interacciones. Particularmente, en aquellas en las que la falta de consistencia aparece comúnmente como, por ejemplo, en el caso de la confusión espontánea de nombres para referirse a un objeto a causa de la fatiga o el paso del tiempo entre que se generó el precedente referencial y el momento en que se volvió a ocupar.

Finalmente, en nuestro estudio los oyentes recibieron instrucciones pre-grabadas frente a las cuales ellos no podían responder. Esto podría generar ciertas diferencias en la manera de adaptarse a las características de los hablantes; adaptación que en cualquier caso sería aún mayor, dada la interactividad de la situación (Brennan \& Hanna, 2009). En este contexto, el estudio de los distintos niveles de interactividad plantea la pregunta por otros tipos de comunicación interpersonal que son hoy en día tan comunes como la conversación cara a cara. Un claro ejemplo es la comunicación virtual, en especial la basada en texto. Existe una incipiente línea de investigación desde la psicolingüística, la psicología y la neurociencia cognitiva que busca entender cómo se establece la comprensión en interacciones sin las claves contextuales propias de la conversación, como las expresiones faciales, la dirección de la mirada o la prosodia (Aldunate \& González-Ibáñez, 2017). Esta ausencia de claves contextuales genera una mayor proclividad a la ambigüedad del lenguaje y, por lo tanto, es de relevancia entender en qué medida las expectativas pragmáticas juegan un rol en disminuir esta ambigüedad.

\section{CONCLUSIÓN}

Para poder resolver la referencia conversacional es necesario ir más allá del significado convencional de las palabras e integrar información y claves contextuales 
que disminuyan la ambigüedad del lenguaje. Una importante fuente de información son las expectativas de comportamiento comunicativo que tienen los interlocutores. Entre estas expectativas está la consistencia en el uso de expresiones lingüísticas para referir a una misma entidad repetidamente durante una interacción. El procesamiento del lenguaje en el diálogo es sensible a esta expectativa y es capaz de adaptarse a ella de manera rápida y eficiente. En conclusión, la ambigüedad propia del lenguaje puede ser sobrellevada por nuestra sensibilidad a los patrones generales de comportamiento durante un diálogo y, en particular, la consistencia.

\section{REFERENCIAS BIBLIOGRÁFICAS}

Aldunate, N. \& González-Ibáñez, R. (2017). An integrated review of emoticons in computer-mediated communication. Frontiers in psychology, 7, 2061.

Allopenna, P. D., Magnuson, J. S. \& Tanenhaus, M. K. (1998). Tracking the time course of spoken word recognition using eye movements: Evidence for continuous mapping models. Journal of memory and language, 38(4), 419-439.

Barr, D. J. \& Keysar, B. (2002). Anchoring comprehension in linguistic precedents. Journal of Memory and Language, 46(2), 391-418.

Baayen, R. H., Davidson, D. J. \& Bates, D. M. (2008). Mixed-effects modeling with crossed random effects for subjects and items. Journal of memory and language, 59(4), 390-412.

Baron-Cohen, S. (1988). Social and pragmatic deficits in autism: Cognitive or affective?. Journal of autism and developmental disorders, 18(3), 379-402.

Bates, D., Maechler, M., Bolker, B. \& Walker, S. (2014). lme4: Linear mixed-effects models using Eigen and S4. R package version, 1(7), 1-23.

Brennan, S. E. \& Clark, H. H. (1996). Conceptual pacts and lexical choice in conversation. Journal of Experimental Psychology: Learning, Memory, and Cognition, 22(6), 1482.

Brennan, S. E. \& Hanna, J. E. (2009). Partner-specific adaptation in dialog. Topics in Cognitive Science, 1(2), 274-291.

Brennan, S. E. \& Hanna, J. E. (2017). Psycholinguistic approaches. En E. Weigand (Ed.), The Routledge Handbook of Language and Dialogue (pp. 93-108). Nueva York: Routledge.

Brennan, S. E., Galati, A. \& Kuhlen, A. K. (2010). Two minds, one dialog: Coordinating speaking and understanding. Psychology of Learning and Motivation, 53, 301-344. 
Brown-Schmidt, S. (2009). Partner-specific interpretation of maintained referential precedents during interactive dialog. Journal of memory and language, 61(2), 171190.

Carmichael, L., Hogan, H. P. \& Walter, A. A. (1932). An experimental study of the effect of language on the reproduction of visually perceived form. Journal of experimental Psychology, 15(1), 73.

Chafe, W. L. (1976). Givenness, contrastiveness, definiteness, subjects, topics, and point of view. En C. N. Li (Ed.), Subject and topic. Nueva York: Academic Press.

Cornish, F. (2008). How indexicals function in texts: Discourse, text, and one neoGricean account of indexical reference. Journal of Pragmatics, 40(6), 997-1018.

Clark, H. H. (1996). Using language. Cambridge University Press.

Clark, H. H. \& Carlson, T. B. (1981). Context for comprehension. Attention and performance IX, 313-330.

Clark, H. H. \& Marshall, C. R. (1981). Definite reference and mutual knowledge. En A. H. Joshe, B. Webber \& I. A. Sag (Eds.), Elements of discourse understanding (pp. 10-63). Cambridge, Inglaterra: Cambridge University Press.

Clark, H. H. \& Wilkes-Gibbs, D. (1986). Referring as a collaborative process. Cognition, 22(1), 1-39.

Clark, H. H. \& Brennan, S. E. (1991). Grounding in communication. Perspectives on socially shared cognition, 13(1991), 127-149.

Enfield, N. J., Stivers, T. \& Levinson, S. C. (2010). Question-response sequences in conversation across ten languages: An introduction. Journal of Pragmatics, 42(10), 2615-2619.

Garrod, S. \& Anderson, A. (1987). Saying what you mean in dialogue: A study in conceptual and semantic co-ordination. Cognition, 27(2), 181-218.

Gernsbacher, M. A. (1989). Mechanisms that improve referential access. Cognition, 32(2), 99 .

Grice, H. P. (1957). Meaning. The philosophical review, 66(3), 377-388.

Grice, H. P. (1975). Logic and conversation. En S. Davis (Ed.), Pragmatics: A reader (pp. 305-315). Nueva York: Oxford University Press.

Hanna, J. E., Tanenhaus, M. K. \& Trueswell, J. C. (2003). The effects of common ground and perspective on domains of referential interpretation. Journal of Memory and Language, 49(1), 43-61. 
Horton, W. S. \& Slaten, D. G. (2012). Anticipating who will say what: The influence of speaker-specific memory associations on reference resolution. Memory \& cognition, 40(1), 113-126.

Huang, Y. (2000). Anaphora: A cross-linguistic approach. Oxford University Press on Demand.

Huang, Y. (2007). Pragmatics (Oxford Textbooks in Linguistics). Oxford: Oxford University Press.

Huettig, F., Rommers, J. \& Meyer, A. S. (2011). Using the visual world paradigm to study language processing: A review and critical evaluation. Acta psychologica, 137(2), 151-171.

Keysar, B., Barr, D. J., Balin, J. A. \& Brauner, J. S. (2000). Taking perspective in conversation: The role of mutual knowledge in comprehension. Psychological Science, 11(1), 32-38.

Knutsen, D. \& Le Bigot, L. (2017). Conceptual match as a determinant of reference reuse in dialogue. Journal of Experimental Psychology: Learning, Memory, and Cognition, 43(3), 350.

Krauss, R. M. \& Weinheimer, S. (1964). Changes in reference phrases as a function of frequency of usage in social interaction: A preliminary study. Psychonomic Science, 1(1-12), 113-114.

Kronmüller, E. M. (2008). Listeners' adaptation to the local and global environments of language use. University of California: Riverside.

Kronmüller, E. \& Barr, D. J. (2007). Perspective-free pragmatics: Broken precedents and the recovery-from-preemption hypothesis. Journal of Memory and Language, 56(3), 436-455.

Kronmüller, E. \& Barr, D. J. (2015). Referential precedents in spoken language comprehension: A review and meta-analysis. Journal of Memory and Language, 83, 1-19.

Kronmüller, E., Morisseau, T. \& Noveck, I. A. (2014). Show me the pragmatic contribution: A developmental investigation of contrastive inference. Journal of child language, 41(5), 985-1014.

Kronmüller, E., Noveck, I., Rivera, N., Jaume, F. \& Barr, D. (2017). The positive side of a negative reference: The delay between linguistic processing and common ground. Royal Society Open Science, 4(2), 160827. 
Levinson, S. C. (2001). Covariation between spatial language and cognition. En M. Bowerman, S. C. Levinson \& S. Levinson (Eds.), Language acquisition and conceptual development (pp. 566-588). Cambridege: Cambridge University Press.

Levinson, S. C. \& Torreira, F. (2015). Timing in turn-taking and its implications for processing models of language. Frontiers in psychology, 6 .

Markman, E. M. \& Wachtel, G. F. (1988). Children's use of mutual exclusivity to constrain the meanings of words. Cognitive psychology, 20(2), 121-157.

Mervis, C. B. \& Bertrand, J. (1994). Acquisition of the novel name-nameless category (N3C) principle. Child development, 65(6), 1646-1662.

Metzing, C. \& Brennan, S. E. (2003). When conceptual pacts are broken: Partnerspecific effects on the comprehension of referring expressions. Journal of Memory and Language, 49(2), 201-213.

Mitkov, R. (2009). Anaphora resolution. En R. Mitkov (Ed.), The Oxford Handbook of Computational Linguistics (pp. 266-283). Oxford: Oxford University Press.

Nieuwland, M. S. \& van Berkum, J. J. (2008). The interplay between semantic and referential aspects of anaphoric noun phrase resolution: Evidence from ERPs. Brain and Language, 106(2), 119-131.

Noveck, I. (2018). Experimental pragmatics: The making of a cognitive science. Cambridge University Press.

Noveck, I. A. \& Sperber, D. (2004). Experimental pragmatics. Basingstoke: Palgrave Macmillan.

Noveck, I. A. \& Reboul, A. (2008). Experimental pragmatics: A Gricean turn in the study of language. Trends in Cognitive Sciences, 12(11), 425-431.

Osterhout, L. \& Mobley, L. A. (1995). Event-related brain potentials elicited by failure to agree. Journal of Memory and language, 34(6), 739-773.

Pyszczynski, T. \& Greenberg, J. (1987). Toward an integration of cognitive and motivational perspectives on social inference: A biased hypothesis-testing model. Advances in experimental social psychology, 20, 297-340.

Shintel, H. \& Keysar, B. (2007). You said it before and you'll say it again: Expectations of consistency in communication. Journal of Experimental Psychology: Learning, Memory, and Cognition, 33(2), 357-369.

Strawson, P. F. (1950). On referring. Mind, 59(235), 320-344.

Sukthanker, R., Poria, S., Cambria, E. \& Thirunavukarasu, R. (2018). Anaphora and Coreference Resolution: A Review. arXiv preprint arXiv:1805.11824. 
Streb, J., Hennighausen, E. \& Rösler, F. (2004). Different anaphoric expressions are investigated by event-related brain potentials. Journal of Psycholinguistic Research, 33(3), 175-201.

Tanenhaus, M. K. \& Trueswell, J. C. (2005). Eye movements as a tool for bridging the language-as-product and language-as-action traditions. Approaches to studying world-situated language use: Bridging the language-as-product and language-action traditions, 3-37.

Tomasello, M., Carpenter, M., Call, J., Behne, T. \& Moll, H. (2005). Understanding and sharing intentions: The origins of cultural cognition. Behavioral and Brain Sciences, 28(5), 675-735.

\section{NOTA}

1Autor correspondiente.

\section{* AGRADECIMIENTOS}

Esta investigación fue financiada por la Comisión Nacional de Investigación Científica y Tecnológica de Chile (CONICYT) a través del proyecto FONDECYT N¹1100226 a Edmundo Kronmüller. 